\title{
Development a Reverse Triage System Based on Modified Sequential Organ Failure Assessment for Increasing the Critical Care Surge Capacity
}

\author{
Abbasali Ebrahimian, Hossein Ghasemian-Nik', Raheb Ghorbani², Ali Fakhr-Movahedi \\ Nursing Care Research Center, Semnan University of Medical Sciences, ${ }^{1}$ Student Research Committee, Nursing and Midwifery school, Semnan University of Medical \\ Sciences, ${ }^{2}$ Social Determinants of Health Research Center, Semnan University of Medical Sciences, Semnan, Iran
}

\section{Abstract}

Context: The capacity completeness are one of the serious problems in the bed's managements of the critical care units in a crisis and disaster situation. Reverse triage can help to hospital surge capacity in this situations. Aims: The aim of this study was to develop a reverse triage system based on Modified Sequential Organ Failure Assessment (MSOFA) for increasing critical care surge capacity. Settings and Design: This study was a prospective design that performed on the medical patients in critical care unit. Subjects and Methods: The MSOFA score for each patient was calculated in admission time and be continued until discharging time from critical care unit. Statistical Analysis Used: The Cox regression method was used to determine the relative risk values. At last, the patients were divided into three levels for reverse triage. Results: Four hundred and twenty patients were participated in this study. The mean of patients' MSOFA scores in the $1^{\text {st }}$ day of admission in Critical Care was $5.40 \pm 3.8$. The relative risk of internal patients discharge from critical care was $(8.2 \%)$. Death relative risks were $<25 \%$, higher than $70 \%$ and between $25.1 \%$ and $69.9 \%$ for three color level of green, black, and red, respectively. Conclusion: The MSOFA scores can contribute to the design a leveling system for discharging patients from critical care unit. Based on this system, the members of the caring team can predict the final health status of the patient.

Keywords: Modified sequential organ failure, reverse triage, surge capacity

\section{INTRODUCTION}

High occupancy rate and capacity completeness are one of the serious problems in managing the beds of the critical care units. ${ }^{[1]}$ In this context, hospital surge capacity is one of the strategies that can introduce a coordination between patients' referrals to hospitals and convenience facilities. ${ }^{[2]}$ Hospital surge capacity means strengthening the potential of the hospital in the face of a crisis or a disaster. In addition, it improves the ability of hospitals when they face an increasing demand for service delivery. ${ }^{[3-6]}$ Reverse triage can help hospital surge capacity. The goal of reverse triage is safe to discharge of hospitalized patients and allocation of hospital resources to patients who need more advanced health-care services. ${ }^{[2,7,8]}$ Early warning scoring systems are one of the methods that can enhance the success of reverse triage. Early warning scoring systems are used to assess the clinical status; estimate the probability of recovery, the physiological uncertainty, and

\begin{tabular}{|l|l|}
\hline \multicolumn{3}{|c|}{ Access this article online } \\
\hline Quick Response Code: & Website: \\
\hline & www.ijccm.org \\
\hline & \\
\hline
\end{tabular}

the probability of survival; and prioritize and optimize patient care. ${ }^{[9,10]}$ These systems can also be used in decision-making and clinical judgment, standardization of research in the field of critical care, determining work pressure, optimal allocation of human and technical resources, and comparing the quality of care between the critical care units. ${ }^{[11,12]}$

Sequential organ failure assessment (SOFA) is one of the well-known scoring systems. This system was developed in 1994 to review the failure of six organs, namely pulmonary,

Address for correspondence: Dr. Ali Fakhr-Movahedi, Nursing Care Research Center, Semnan University of Medical Sciences, Semnan, Iran.

Room \#120, $1^{\text {th }}$ Floor, School of Nursing and Midwifery, Semnan University of Medical Sciences, Km5 Damghan Road, Semnan, Iran. E-mail: alimovi49@semums.ac.ir

This is an open access journal, and articles are distributed under the terms of the Creative Commons Attribution-NonCommercial-ShareAlike 4.0 License, which allows others to remix, tweak, and build upon the work non-commercially, as long as appropriate credit is given and the new creations are licensed under the identical terms.

For reprints contact: reprints@medknow.com

How to cite this article: Ebrahimian A, Ghasemian-Nik H, Ghorbani R, Fakhr-Movahedi A. Development a reverse triage system based on modified sequential organ failure assessment for increasing the critical care surge capacity. Indian J Crit Care Med 2018;22:575-9. 
blood, cardiovascular, liver, central nervous, and renal organs. ${ }^{[13]}$ Then, in 2007, Grissom et al. introduced a simplified version of this system known as the Modified Sequential Failure Organ Assessment (MSOFA). In this version, laboratory tests such as platelet count were removed, and $\mathrm{SpO}_{2} / \mathrm{FiO}_{2}$ ratio was replaced with $\mathrm{PaO}_{2} / \mathrm{FiO}_{2}$. In addition, the evaluation of serum bilirubin level was replaced with clinical evaluation. ${ }^{[14]}$

Various studies have used MSOFA for triage, prognostic measurements, and assessing the mortality rate of patients in critical care settings. ${ }^{[9,15,16]}$ However, so far, this system has not been used as a trusted tool for reverse triage.

Therefore, due to the importance of early discharge of patients and increasing the capacity of the critical care units, and since the researchers did not find an evidence-based method for reverse triage of patients, this study was conducted with the aim of developing a reverse triage system based on MSOFA scores for increasing the critical care surge capacity.

\section{Subjects and Methods}

\section{Design, setting, and participants}

This study was performed using a longitudinal, prospective design from March 2015 to November 2016. Research subjects were hospitalized medical patients in the critical care unit of a referral hospital in Iran. The sample size was determined based on the recommendation of researchers to validate the results of multiple logistic regressions. This recommendation states that the number of completed questionnaires should be between 5 and 10 times of the number of items in the questionnaire. ${ }^{[17]}$ Therefore, since the items of MSOFA systems were 22 variables, the minimum sample size for this study was estimated as 220 patients. However, the researchers obtained 420 patients to achieve more favorable results. The inclusion criteria included the consent of the patient or to participate in the study and the possibility of completing the MSOFA questionnaire for the patient. The exclusion criteria included continuation of the patient's treatment in another center, uncertainty of the patient's outcome after discharge from the critical care unit, and failure to perform necessary laboratory tests to estimate the MSOFA score.

\section{Data collection and measurement}

Data were collected using a demographic questionnaire and the MSOFA system. The demographic questionnaire was applied to collect information about the patients' age, gender, cause, and number of hospitalizations, duration of stay in the critical care unit, and the presence or absence of a chronic disease. The MSOFA system was applied to measure the parameters related to respiratory, cardiovascular, renal, hepatic, and central nervous systems. The function of each organ was scored from 0 to 4 . Then, the scores obtained from the performance of these five vital organs are combined, and the MSOFA score of the patient is determined from 0 to 20 . A higher score indicates a greater deterioration in the patient's condition [Table 1]. The MSOFA system is a valid and reliable tool that has been used in several studies, ${ }^{[14,18,19]}$ as its reliability coefficient for hospitalized patients in the critical care units has been found to be $0.94 .{ }^{[18]}$

The demographic data and the MSOFA score for each patient were estimated and documented at the admission time. The estimation and documentation of MSOFA scores were continued till the discharge time from the critical care unit. To calculate the MSOFA score, the lowest levels of daily variables that indicated the worst conditions of the patient were considered as the patient's score on that particular day. The final status of the patients was monitored till discharge from the hospital. Then, the patients were categorized into two groups according to their final status. The patients in the first group were discharged from the hospital with partial or complete improvement in their health status. However, the second group included dead patients or patients who had returned to the critical care unit before discharge from the hospital. The final status of the patients was used as a criterion for statistical tests.

\section{Statistical analysis}

Data analysis was performed using the SPSS software, edition 16 (IBM Corporation). The Cox regression method (Backward: Wald method) was used to determine the relative risk values. The leveling of the transmission risk of patients from the critical care unit was carried out in two steps. In the first step, the relative frequency of death or the return of patients to the critical care unit was determined based on their MSOFA scores at the discharge time. Accordingly, the MSOFA scores were divided into the following three categories: the scores of patients with $<4 \%$ mortality or return to the critical care unit, the scores of patients with 4\%-99\% mortality or return to the critical care unit, and the scores of patients with $100 \%$ mortality.

\begin{tabular}{|c|c|c|c|c|c|}
\hline Organ system & 0 & 1 & 2 & 3 & 4 \\
\hline $\begin{array}{l}\text { Respiratory } \mathrm{SpO}_{2} / \mathrm{FiO}_{2} \\
\text { Liver }\end{array}$ & $\begin{array}{l}>400 \\
\text { No scleral icterus or jaundice }\end{array}$ & $\leq 400$ & $\leq 315$ & $\begin{array}{l}\leq 235 \\
\text { Scleral icterus or jaundice }\end{array}$ & $\leq 150$ \\
\hline $\begin{array}{l}\text { Cardiovascular, } \\
\text { hypotension }\end{array}$ & No hypotension & $\mathrm{MAP}<70 \mathrm{mmHg}$ & $\begin{array}{l}\text { Dopamine } \leq 5 \\
\text { or dobutamine } \\
\text { any dose }\end{array}$ & $\begin{array}{l}\text { Dopamine }>5 \\
\text { Epinephrine } \leq 0.1 \\
\text { Norepinephrine } \leq 0.1\end{array}$ & $\begin{array}{l}\text { Dopamine }>15 \\
\text { Epinephrine }>0.1 \\
\text { Norepinephrine }>0.1\end{array}$ \\
\hline CNS, Glasgow coma scale & 15 & $13-14$ & $10-12$ & $6-9$ & $<6$ \\
\hline Renal, creatinine $\mathrm{mg} / \mathrm{dl}$ & $<1.2$ & $1.2-1.9$ & $2.0-3.4$ & $3.5-4.9$ & $>5.0$ \\
\hline
\end{tabular}

MAP: Mean arterial pressure; CNS: Central nervous systems 
In the second step, the relative risk calculated using Cox regression was multiplied by MSOFA scores. Using this method, the relative risk of death after discharge was obtained based on the MSOFA scores. Then, the relative risk values based on MSOFA scoring, the relative risk of death, and patients' return to the critical care unit were put together in a table. In this manner, the patients were divided into the following three levels for discharge: First (green color), second (black color), and third (red color). The significance level was considered to be 0.05 in all statistical calculations.

\section{Ethical considerations}

This study was approved by the Research Ethics Committee of Semnan University of Medical Sciences, Semnan, Iran (No: IR. SEMUMS. REC.1394.182). In addition, informed consent was obtained from all participants, and the purpose of the study was explained to each patient. It was also emphasized to the patients that their participation in the study is voluntary and that they could withdraw from the study at any time.

\section{RESULTS}

Of the study patients, $234(55.7 \%)$ were males and the remaining were females. The average age of the patients was $42.91 \pm 20.64$ years. In addition, 274 patients $(65.2 \%)$ had a history of hospitalization in the critical care unit. The average length of stay in the critical care unit was $6.8 \pm 4.88$ days, and 243 patients $(57.9 \%)$ were attached to a mechanical ventilation device during the critical care unit admission. Of the patients, $296(70.5 \%)$ were discharged from the hospital with relative improvement, 9 (2.1\%) again needed special care and were readmitted to the critical care unit, and 115 patients $(27.4 \%)$ died before discharge from the hospital [Table 2].

The mean MSOFA score of the patients on the $1^{\text {st }}$ day of admission in the critical care unit was $5.4 \pm 3.8$. With the increase in the number of admission days, the mean MSOFA scores were increased, from to $6.1 \pm 4.3$ and $10.8 \pm 3.8$ on the

\begin{tabular}{lc}
\hline \multicolumn{2}{l}{ Table 2: Demographic variables and hospitalization data } \\
\hline Variables & Frequency \\
\hline Age (year) & $42.91 \pm 20.64$ \\
The average length of stay in critical care unit (day) & $6.80 \pm 4.88$ \\
Critical care unit hospitalization history, $n(\%)$ & \\
Yes & $274(65.2)$ \\
No & $146(34.8)$ \\
Gender, $n(\%)$ & \\
Male & $234(55.7)$ \\
Female & $186(44.3)$ \\
Use mechanical ventilation, $n(\%)$ & \\
Yes & $16(10.4)$ \\
No & $81(52.9)$ \\
Outcome of patients, $n(\%)$ & \\
Discharge from hospital & $296(70.5)$ \\
Re-admission to critical care unit & $9(2.1)$ \\
Death in hospital & $115(27.4)$ \\
\hline${ }^{a}$ Data are presented as $n(\%)$ or Mean \pm SD. SD: Standard deviation
\end{tabular}

$10^{\text {th }}$ and the $20^{\text {th }}$ days of admission, respectively. The relative risk of patients' transmission from the critical care unit was 1.082 (95\% CI=1.062-1.099). The calculated relative risk showed that for each unit increase in MSOFA scores, the risk of death after discharge from the critical care unit or readmission to the critical care unit was increased by $8.2 \%$.

The results showed that only $3 \%$ of discharged patients with an MSOFA score of $\leq 3$ returned to the critical care unit and all patients with an MSOFA score of $\geq 9$ died. Furthermore, $8 \%-66.6 \%$ of patients died or returned to the critical care unit, with MSOFA scores ranging from 4 to 8 . Finally, by multiplying the MSOFA scores with the relative risk value of death after discharge, the relative risk $(8.2 \%)$ of patients discharged from the critical care unit was calculated based on each MSOFA score. By combining the MSOFA scores, the numerical values of relative risk based on each score, and the mortality rate in each score, the leveling of the transmission risk of medical patients from the critical care unit was obtained. The relative risks of death were $<25 \%,>70 \%$, and between $25.1 \%$ and $69.9 \%$ for the three color levels of green, black, and red, respectively [Table 3 ].

\section{Discussion}

Triage protocols are designed to provide maximum benefit to a large number of patients. ${ }^{[15]}$ In this study, a protocol was developed to increase hospital surge capacity in the critical care unit. Therefore, the MSOFA system was used as a tool for predicting the patients' deterioration for leveling of the transmission risk of medical patients from the critical care unit. In this context, several studies have demonstrated that MSOFA has good power in predicting morbidity and mortality of patients. ${ }^{[14,19-21]}$

The results of this study showed that the average MSOFA score of medical patients at the admission time in the critical care unit was $5.4 \pm 3.8$. Sendagire et al. and Halim et al. reported the average MSOFA scores on the $1^{\text {st }}$ day of admission to be $6.3 \pm 5.7$ and $3.1 \pm 98.95$, respectively. ${ }^{[19,20]}$ This finding suggests that different approaches were used when deciding on patients' admission in the critical care unit. It appears that factors such as the level of development of medical knowledge, the availability of advanced medical equipment, and the per capita number of critical care unit beds of a country are effective in determining patients' hospitalization in the critical care units. Therefore, the results of this study can be used to equalize and provide evidence for using the MSOFA system.

In the present study, the mean MSOFA scores were found to be increased according to the increase in the number of admission days. Similarly, Gholipour et al. and Grissom et al. reported that the mean MSOFA scores showed an increasing trend over time in patients admitted to the critical care unit. ${ }^{[14,21]}$ However, Sendagire et al. reported a decreasing trend. ${ }^{[19]}$ The reason for the increase in the mean MSOFA scores in the current study and some other studies is that over time, some patients who were better and had lower MSOFA scores were discharged 


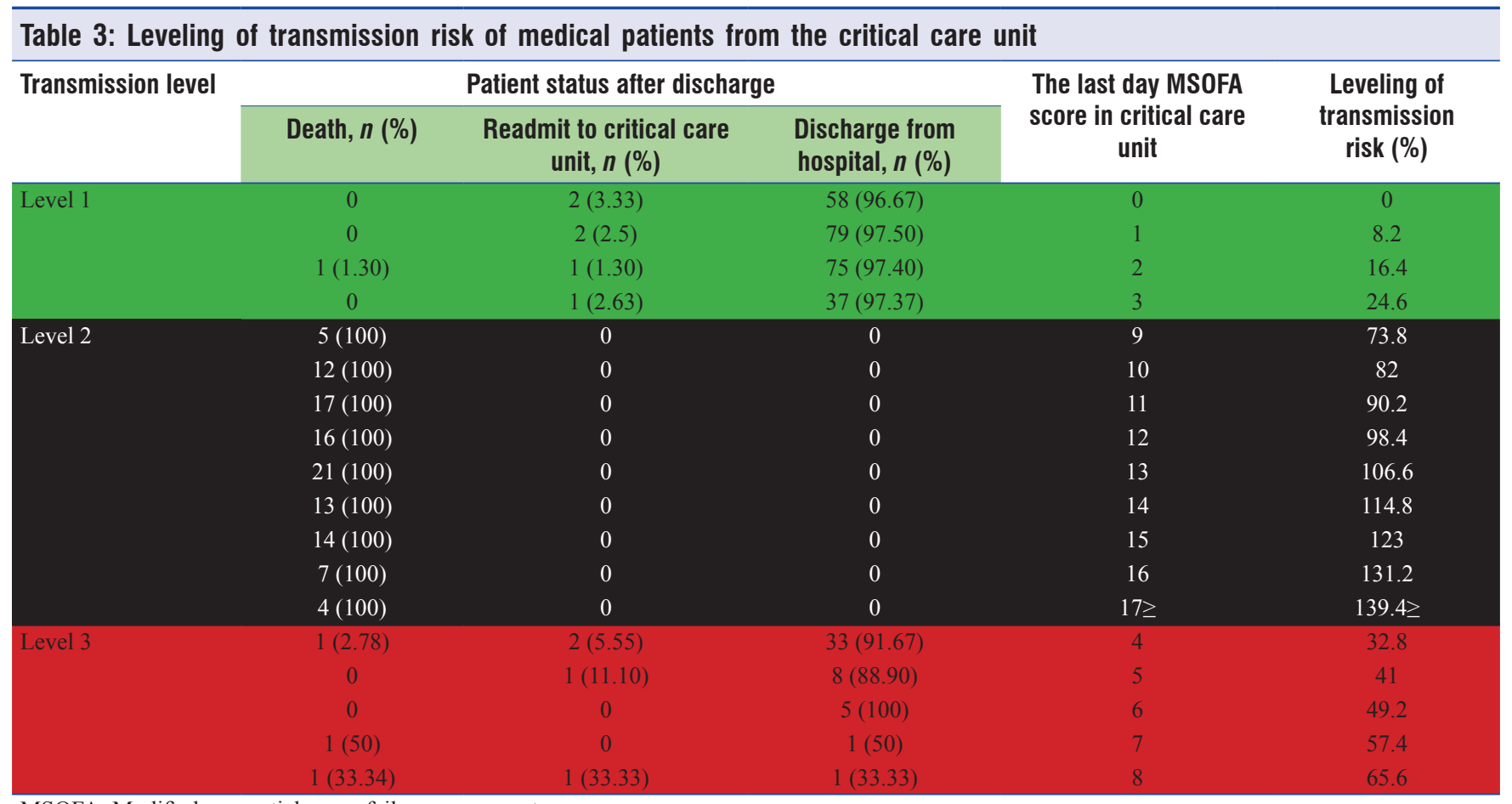

MSOFA: Modified sequential organ failure assessment

from the critical care unit. Moreover, the death of patients with high MSOFA scores in the first few days of admission was one of the reasons for the increase in the mean scores. ${ }^{[18]}$ Therefore, the trend observed in the MSOFA scores may be an indicator of the advancement of medical systems. In fact, the decreasing trend of MSOFA scores may be because of the following factors: Late referral to hospitals due to lack of diagnostic and care facilities, lack of vacant beds in the critical care units, and death in the early days of admission in the critical care units.

In this study, the relative risk of patients' transmission from the critical care units was $1.082(95 \% \mathrm{CI}=1.062-1.099)$, which indicated that for each unit increase in the MSOFA scores, the risk of death after discharge or readmission to the critical care unit would increase by $8.2 \%$. In this context, although there was no study regarding the relative risk assessment of SOFA scores at the time of admission or discharge of patients, several studies have used the odds ratio to determine the risk of MSOFA scores. In the study of Babamohamadi et al., the odds ratio was calculated as 1.325 . This value indicated that for each unit increase in the MSOFA score, the probability of death increased by $32 \%{ }^{[18]}$ Namendys-Silva et al. reported that the probability of patients' death was $35 \%$ for each unit increase in MEXSOFA scores. ${ }^{[22]}$ The relative risk indicates a more accurate estimate of the probable risk exposure compared with the odds ratio. Therefore, the use of relative risk rather than the odds ratio can prevent-related issues and biases. ${ }^{[23]}$

In this study, the odds ratio was used to increase the accuracy and precision of the calculation of risk of death in patients. Therefore, compared with other studies, the risk level for each unit increase in MSOFA scores was lower than the reported level in other studies.

In this study, the leveling of the risk of patients' discharge from the critical care unit led to the establishment of a three-level protocol for reverse triage. These three levels were related to the relative risk of death. In the first level, patients had the lowest chance of death or return to the special department. The second level was pertained to patients who were in the final stage of life, and no improvement in health status was assumed for them. Usually, these patients were transferred to the hospital departments. However, in the third level, there were patients who could benefit from critical care. Therefore, it is more cost-effective to consider the major care measures for these patients. In fact, according to the results of this study, these patients had the highest priority in accessing the critical care. Christian et al. used SOFA to design triage protocols for use in avian influenza crises. They used the review literature process, internet search, and consultation with the critical care intensivist to design their protocol. Finally, they prioritized the patients into four levels. ${ }^{\left[{ }^{[5]}\right.}$ Kelen et al. identified the criteria for early discharge during disasters using a specialized panel. They divided the patients according to a scale of 10 scores into the following three importance groups: importance (7-10), moderate importance $(5-5)$, and low importance $(<4) .^{[7]}$ According to the above-mentioned studies, the specialized panel and the literature were used for leveling the patients' status. In addition, in most of the studies, a triage protocol was designed to prioritize the admission of patients in the critical care units. However, in the present study, the proposed triage protocol was based on data that were collected from patients admitted to the critical care units and a reverse triage protocol. 


\section{Conclusion}

MSOFA can be used as a deciding tool in discharging patients from the critical care unit. In this study, MSOFA contributed to designing a leveling system for patients admitted to the critical care unit. Based on this system, caring team members can predict the final health status of the patient. Therefore, to obtain more scientific evidence, it is recommended that this system should be used to assess the risk of transmission of patients with other clinical conditions in the critical care units.

\section{Financial support and sponsorship}

This study was supported by Semnan University of Medical Sciences.

\section{Conflicts of interest}

There are no conflicts of interest.

\section{References}

1. Kim SH, Chan CW, Olivares M, Escobar G. ICU admission control: An empirical study of capacity allocation and its implication for patient outcomes. Manag Sci 2014;61:19-38.

2. Kelen GD, Kraus CK, McCarthy ML, Bass E, Hsu EB, Li G, et al. Inpatient disposition classification for the creation of hospital surge capacity: A multiphase study. Lancet 2006;368:1984-90.

3. Watson SK, Rudge JW, Coker R. Health systems" "surge capacity": State of the art and priorities for future research. Milbank Q 2013;91:78-122.

4. Adams LM. Exploring the concept of surge capacity. Online J Issues Nurs 2009;14:1-12.

5. Kearns RD, Cairns BA, Cairns CB. Surge capacity and capability. A review of the history and where the science is today regarding surge capacity during a mass casualty disaster. Front Public Health 2014;2:29.

6. Bayram JD, Sauer LM, Catlett C, Levin S, Cole G, Kirsch TD, et al. Critical resources for hospital surge capacity: An expert consensus panel. PLoS Curr 2013;5. pii: ecurrents.dis. 67c1afe8d78ac2ab0ea52319eb119688.

7. Kelen GD, McCarthy ML, Kraus CK, Ding R, Hsu EB, Li G, et al. Creation of surge capacity by early discharge of hospitalized patients at low risk for untoward events. Disaster Med Public Health Prep 2009;3:S10-6.

8. Frolic A, Kata A, Kraus P. Development of a critical care triage protocol for pandemic influenza: Integrating ethics, evidence and effectiveness. Healthc Q 2009;12:54-62.

9. Ebrahimian A, Fakhr-Movahedi A, Ghasemian-Nik H. Evaluation the mean alteration trends and validation of nine equivalents of nursing manpower use score in medical patients of Intensive Care Units. Koomesh 2017;19:458-65.

10. Asad Zandi M, Karati T, Tadrisi SD, Ebadi A. Estimation of the mortality rate using the APACHE II standard disease severity scoring system in Intensive Care Unit patients. J Crit Care Nurs 2012;4:209-14.

11. Schusterschitz N, Joannidis M. Predictive capacity of severity scoring systems in the ICU. Acute Kidney Injury. Contrib Nephrol. Basel, Karger, 2007. p. 92-100.

12. Ebrahimian A, Masoumi G, Jamshidi-Orak R, Seyedin H. Development and psychometric evaluation of the pre-hospital medical emergencies early warning scale. Indian J Crit Care Med 2017;21:205-12.

13. Rapsang AG, Shyam DC. Scoring systems in the intensive care unit: A compendium. Indian J Crit Care Med 2014;18:220-8.

14. Grissom CK, Brown SM, Kuttler KG, Boltax JP, Jones J, Jephson AR, et al. A modified sequential organ failure assessment score for critical care triage. Disaster Med Public Health Prep 2010;4:277-84.

15. Christian MD, Hawryluck L, Wax RS, Cook T, Lazar NM, Herridge MS, et al. Development of a triage protocol for critical care during an influenza pandemic. CMAJ 2006;175:1377-81.

16. Moreno R, Miranda DR, Matos R, Fevereiro T. Mortality after discharge from intensive care: The impact of organ system failure and nursing workload use at discharge. Intensive Care Med 2001;27:999-1004.

17. Geoffrey RN, David LS. Biostatistics. $2^{\text {nd }}$ ed. London: Hamilton; 2002.

18. Babamohamadi H, Ebrahimian A, Paknazar F, Torkamandi H. Clinical effectiveness of modified sequential organ failure assessment scoring system for predicting ICU indexing scores. Tehran Univ Med J 2016;74:509-16.

19. Sendagire C, Lipnick MS, Kizito S, Kruisselbrink R, Obua D, Ejoku J, et al. Feasibility of the modified sequential organ function assessment score in a resource-constrained setting: A prospective observational study. BMC Anesthesiol 2017;17:12.

20. Halim DA, Murni TW, Redjeki IS.Comparisonof Apache II, SOFA, and modified SOFA scores in predicting mortality of surgical patients in intensive care unit at Dr. Hasan Sadikin General Hospital. Crit Care Shock 2009;12:157-69.

21. Gholipour Baradari A, Sharifi H, Firouzian A, Daneshiyan M, Aarabi M, Talebiyan Kiakolaye Y, et al. Comparison of proposed modified and original sequential organ failure assessment scores in predicting ICU mortality: A prospective, observational, follow-up study. Scientifica (Cairo) 2016;2016:7379325.

22. Namendys-Silva SA, González-Herrera MO, García-Guillén FJ, Texcocano-Becerra J, Herrera-GómezA. Outcome of critically ill patients with hematological malignancies. Ann Hematol 2013;92:699-705.

23. Robbins AS, Chao SY, Fonseca VP. What's the relative risk? A method to directly estimate risk ratios in cohort studies of common outcomes. Ann Epidemiol 2002;12:452-4. 